Cahiers Société

\title{
L'éternelle saison des amours : la soumission virtuelle de la pratique sociale au capital
}

\author{
Éric N. Duhaime
}

Numéro 2, décembre 2020

Marx, critique du capital et de la société

URI : https://id.erudit.org/iderudit/1075554ar

DOI : https://doi.org/10.7202/1075554ar

Aller au sommaire du numéro

Éditeur(s)

Collectif Société

ISSN

2562-5373 (imprimé)

2562-5381 (numérique)

Découvrir la revue

Citer cet article

Duhaime, É. N. (2020). L'éternelle saison des amours : la soumission virtuelle de la pratique sociale au capital. Cahiers Société, (2), 71-96.

https://doi.org/10.7202/1075554ar
Résumé de l'article

Cet article vise à problématiser les enjeux qui se rattachent au rôle de plus en plus central que jouent la science et la technologie dans l'économie. Développant notre réflexion à partir de l'oeuvre de Marx, nous cherchons d'abord à rendre compte des concepts de soumission formelle et de soumission réelle qu'il développa à son époque. En tenant compte du passage du capitalisme industriel au capitalisme avancé, nous proposons ensuite le concept de soumission virtuelle de la pratique sociale au capital afin de problématiser les enjeux liés à une nouvelle stratégie monopolistique qui fut mise en place par de grandes corporations au milieu du $\mathrm{XX}^{\mathrm{e}}$ siècle et qui conjugue la R-D et le marketing, c'est-à-dire la production et la

commercialisation d'inventions brevetées. Il en résulte alors un contrôle sur la détermination des objets d'usage futurs qui viendront prendre place dans nos sociétés, lequel renvoie précisément au concept de soumission virtuelle de la pratique sociale au capital. 


\title{
L'éternelle saison des amours : la soumission virtuelle de la pratique sociale au capital
}

\author{
Éric N. DUHAIME \\ Université du Québec à Montréal
}

En s'inspirant de l'œuvre de Marx et, plus spécifiquement, de la notion d'« intellect général ${ }^{1} »$, des auteurs contemporains comme Michael Hardt, Antonio Negri, Yann Moulier Boutang, Carlo Vercellone ou André Gorz ont cherché à problématiser l'avènement d'une économie qui repose de plus en plus sur l'innovation et sur le développement de la science et de la technologie ${ }^{2}$. Pour ces auteurs regroupés autour de la revue Multitudes, cette transformation de l'économie marque un tournant majeur dans l'histoire du capitalisme. En étendant son emprise sur la science et la technologie, voire sur les relations sociales elles-mêmes, l'économie emprunterait désormais une forme « immatérielle ». Nous assisterions ainsi à la mise en place d'une nouvelle phase du développement du capitalisme, soit l'avènement d'un capitalisme « cognitif». Or, cette nouveauté comporte à leurs yeux un potentiel émancipateur qui serait annonciateur de la fin et du dépassement du capitalisme lui-même.

En effet, l'intégration de la production scientifique et technologique saperait les fondements mêmes sur lesquels repose le capitalisme. D'un côté, les résultats immatériels de la production seraient de plus en plus difficilement appropriables puisque la connaissance et les contenus audiovisuels peuvent être reproduits à l'envi et sans effort. De l'autre, la frontière entre le temps de travail et le temps de la vie aurait tendance à se brouiller puisque la production de nouvelles idées constitue une occupation qu'emporte le travailleur avec lui jusqu'à son domicile et dans ses temps libres. En somme, c'est la valeur elle-même qui serait remise en question, telle que la définit Marx, c'est-à-dire comme «temps de travail socialement nécessaire », et qui constitue la médiation centrale du capitalisme et la mesure spécifique de la

\footnotetext{
${ }^{1}$ La notion, en anglais dans le texte original allemand, provient d'un court texte communément désigné sous le nom de «Fragment sur les machines », que l'on retrouve dans Karl Marx, Manuscrits de 18571858 ("Grundrisse »), t. 2, trad. J.-P. Lefebvre, Paris, Éditions sociales, 1980, p. 182-200.

2 Michael Hardt et Antonio Negri, Empire, trad. D.-A. Canal, Paris, Exil, 2000 ; Multitude. Guerre et démocratie à l'âge de l'Empire, trad. N. Guilhot, Paris, La Découverte, 2004 ; Yann Moulier Boutang, Le capitalisme cognitif. La nouvelle grande transformation, Paris, Amsterdam, 2008 ; Carlo Vercellone, «Transformation de la division du travail et general intellect», dans Sommes-nous sorti du capitalisme industriel ?, dir. Carlo Vercellone, Paris, La Dispute, 2003 ; André Gorz, L'immatériel. Connaissance, valeur et capital, Paris, Galilée, 2003.
} 
richesse dans ce mode de production. Pour un auteur comme André Gorz, l'avènement du capitalisme cognitif correspond ainsi au chant du cygne du capitalisme : « Il n'est pas un capitalisme en crise, il est la crise du capitalisme qui ébranle la société dans ses profondeurs 3 ».

Chez Marx, pourtant, la nature et le contenu du travail ne changent rien à sa participation ou non à la valorisation du capital, ce qui tend à invalider la thèse centrale de ces auteurs, à savoir que le développement du travail immatériel serait porteur d'émancipation et annoncerait le dépassement du capitalisme. En effet, c'est ce que Marx précise avec la notion de «travail productif». Contre une certaine tradition de pensée en économie politique qui tend à départager le caractère productif ou improductif du travail sur la base des différents types qu'il peut emprunter, par exemple comme travail manuel ou intellectuel, Marx soutient qu'avec le mode de production capitaliste, compte tenu de la finalité qui lui est spécifique et qui vise l'accumulation de valeur, tout travail est productif s'il participe à la valorisation du capital4. Ainsi, qu'il soit question de travail manuel effectué au contact de la matière et des instruments ou encore d'un travail intellectuel qui consiste à organiser le travail des autres ou à fabriquer des machines, cela ne change absolument rien à l'affaire pour Marx. Le travail d'une personne est productif s'il participe, de concert avec celui des autres, à l'ensemble du procès de production qui permet de valoriser le capital. Ainsi, que le travail emprunte désormais une forme «immatérielle» comme le soutiennent les auteurs cités, cela n'en fait pas davantage un vecteur d'émancipation et de dépassement du capitalisme, celui-ci pouvant tout autant participer à la valorisation du capital que le travail « matériel ».

Le présent article vise ainsi à offrir une interprétation différente et une critique alternative de l'intégration de la production scientifique et technologique au mode de production capitaliste. Pour ce faire, il comporte avant tout une proposition conceptuelle. En nous inspirant également des travaux de Marx, nous proposons le concept de " soumission virtuelle » de la pratique sociale au capital afin de rendre compte et de problématiser les enjeux qui se rattachent au rôle de plus en plus central que jouent la science et la technologie dans l'économie. Il s'agit d'un concept qui prend le contrepied de l'approche soutenue par les auteurs de la revue Multitudes. Ce concept fait évidemment écho à ceux de « soumission formelle » et de « soumission réelle » développés par Marx lui-même et se comprend en étroite relation avec ceux-ci. Pour le dire d'un mot, si la soumission formelle renvoie à l'intégration de pratiques sociales préexistantes au mode de production capitaliste, et que la soumission réelle renvoie aux bouleversements organisationnels et techniques des pratiques sociales intégrées, la

\footnotetext{
3 André Gorz, L'immatériel, op. cit., p. 81-82.

4 Sur la notion de travail productif, voir Karl Marx, Le Capital, livre 1 : Le procès de production du capital, trad. J.-P. Lefebvre, Paris, Presses Universitaires de France, 1993, p. 569-571 ; et aussi Karl Marx, Un chapitre inédit du « Capital », trad. R. Dangeville, Paris, Union Générale d'Éditions, 1971, p. 224.
} 
soumission virtuelle, qui repose sur l'intégration des pratiques scientifiques et technologiques au mode de production capitaliste, renvoie quant à elle à la création des nouveaux objets d'usage qui viendront prendre place au sein des sociétés contemporaines, et donc également aux besoins et aux pratiques de production et de consommation qui s'y rattachent.

\section{Volonté et conscience de l'agir humain}

Pour commencer, nous aimerions insister sur deux aspects fondamentaux de la pensée de Marx qui touchent, au niveau ontologique, à la façon dont il conçoit le mode d'existence des êtres humains dans le cadre de sa philosophie de l'agir. En comparaison aux activités des autres espèces, la spécificité de l'activité humaine relève pour Marx de la « volonté » et de la « conscience ». Dès ses écrits de jeunesse, notamment dans les Manuscrits de 1844, Marx remarque : «L'animal est immédiatement uni à son activité vitale. Il ne s'en différencie pas. Il l'est. L'homme fait de son activité vitale elle-même l'objet de sa volonté et de sa conscience. Il a de l'activité vitale consciente5 ». Le premier aspect, à savoir que l'activité humaine relève de la volonté, renvoie pour Marx au rapport qu'entretiennent les êtres humains envers la nature et qui correspond à ses yeux à un "métabolisme». En effet, en opposition à une large tradition de la pensée occidentale, plutôt que de concevoir les êtres humains comme des êtres séparés de la nature, comme des êtres de culture qui se rapporteraient à la nature comme à un objet extérieur de connaissance ou de maîtrise technique, les êtres humains sont eux-mêmes posés par Marx comme des êtres naturels, c'est-à-dire comme une partie intégrante de la nature. Tout comme les animaux, les êtres humains sont pour lui, d'un côté, des êtres passifs, c'est-à-dire dépendants de la nature environnante afin de satisfaire leurs besoins : respirer, manger, boire, s'abriter, etc. D'un autre côté, les êtres humains sont également des êtres actifs qui, afin de satisfaire leurs besoins, mobilisent des forces qui sont elles-mêmes posées comme étant naturelles, soit leurs appareils sensoriels ainsi que leurs systèmes nerveux et musculaire, dont la coordination leur permet de se déplacer au sein de l'environnement et d'y puiser ce qui est nécessaire à leur subsistance ${ }^{6}$. En ce sens, les êtres humains entretiennent pour Marx un métabolisme constant avec la nature, celle-ci constituant la condition de leur activité et de la satisfaction de leurs besoins. C'est pourquoi, dans Le Capital, le travail s'y trouve défini comme un «procès métabolique » :

Le travail est d'abord un procès qui se passe entre l'homme et la nature, un procès dans lequel l'homme règle et contrôle son métabolisme avec la nature par la médiation de sa propre action. Il se présente face à la matière naturelle comme une puissance

\footnotetext{
5 Karl Marx, Manuscrits économico-philosophiques de 1844, trad. F. Fischbach, Paris, Vrin, 2007, p. 123 ; nous soulignons.

6 Ibid., p. 166.
} 
naturelle lui-même. Il met en mouvement les forces naturelles de sa personne physique, ses bras et ses jambes, sa tête et ses mains pour s'approprier la matière naturelle sous une forme utile à sa propre vie7.

Cela dit, pour Marx, ce procès métabolique ne relève pas chez l'être humain du simple comportement instinctuel, comme chez l'animal ${ }^{8}$. Comme on le voit à la citation précédente, le procès métabolique se trouve plutôt structuré et régulé en fonction de la « médiation » de son action, c'est-à-dire en fonction des formes qu'il confère historiquement à son activité et qu'il assume subjectivement.

Voilà ce que Marx développe davantage en s'intéressant aux cinq moments formels du procès de travail, de même qu'aux formes particulières qu'il emprunte comme ensemble de travaux utiles et concrets. Ces différents moments formels se déclinent de la manière suivante :

1. L'idée, qui constitue l'image mentale ou la représentation a priori des propriétés matérielles et formelles de l'objet que l'on cherche à produire en tant qu'il est destiné à un usage social particulier.

2. Les opérations, soit l'ensemble des manipulations réalisées sur la matière au moyen des instruments.

3. Les moyens, c'est-à-dire les instruments dont le maniement et les propriétés mécaniques et physiques permettent de conférer une forme désirée à la matière.

4. L'objet, soit la matière qui est mise en forme.

5. Le résultat, qui constitue la matérialisation effective de l'idée visée qui emprunte désormais une forme objective, matérielle et tangible9.

Si tout procès de travail comporte selon Marx l'ensemble de ces moments formels, les formes particulières sous lesquelles il s'incarne comme travaux utiles et concrets

\footnotetext{
7 Karl Marx, Le Capital, livre 1, op. cit., p. 199 ; nous soulignons.

${ }^{8}$ La spécificité de la vie humaine chez Marx se trouve en grande partie définie sur le dos de la vie animale, la compréhension qu'il offre de cette dernière se révélant par ailleurs très pauvre, particulièrement limitée en ce qui concerne la reconnaissance d'une dimension intentionnelle aux comportements animaux. Les développements de l'éthologie ne permettent plus aujourd'hui de réduire de la sorte les comportements animaux au seul instinct. Sur cette question et sur la reconnaissance d'une dimension réflexive à la vie animale développée dans la lignée des travaux de Michel Freitag, voir: Éric Duhaime, Le génie génétique : la privatisation du vivant au sein du capitalisme avancé, mémoire, Université du Québec à Montréal, 2009.

9 Si, à la page 200 de l'édition citée, Marx ne mentionne explicitement que trois moments, soit « l'activité adéquate à une fin, ou encore le travail proprement dit, son objet, et son moyen », c'est bien de cinq moments dont il est question plus tôt dans l'ouvrage alors qu'il aborde le concept de travail utile : «L'habit est une valeur d'usage qui satisfait un besoin particulier. Pour le faire, il faut une espèce déterminée d'activité productive. Celle-ci est déterminée par sa finalité, sa façon d'opérer, son objet, ses moyens et son résultat » (Karl Marx, Le Capital, livre 1, op. cit., p. 47 ; nous soulignons).
} 
supposent toutefois une détermination particulière des moments qui en sont constitutifs, ainsi que leur organisation et leur articulation sous une forme spécifique. À cet égard, Marx insiste sur la centralité du moment que constitue l'idée qui agit à la manière d'une finalité en fonction de laquelle se trouvent structurés les autres moments du procès de travail. De fait, il s'agit là précisément de ce qui permet pour lui de distinguer l'activité humaine du comportement animal :

ce qui distingue d'emblée le plus mauvais architecte de la meilleure abeille, c'est qu'il a construit la cellule dans sa tête avant de la construire dans la cire. Le résultat auquel aboutit le procès de travail était déjà au commencement dans l'imagination du travailleur, existait donc déjà en idée. Non pas qu'il effectue simplement une modification dans la forme de la réalité naturelle : il y réalise en même temps son propre but, qu'il connaît, qui détermine comme une loi la modalité de son action, et auquel il doit subordonner sa volonté10.

C'est pourquoi, d'un point de vue particulier, tout procès de travail utile constitue pour Marx une activité orientée en finalité ou encore, comme il le dit lui-même, une " activité adéquate à une finn"11. En tant que représentation mentale de l'objet que l'on cherche à produire, l'idée agit comme une finalité à laquelle il faut «subordonner sa volonté ». Ainsi, les formes particulières sous lesquelles s'incarne historiquement l'activité humaine relèvent de la volonté et sont donc subjectivement assumées par les individus qui les réalisent dans le cadre de sociétés déterminées.

Le second aspect sur lequel nous aimerions insister renvoie à la dimension consciente de l'activité humaine. Il s'agit là d'un autre aspect qui permet à Marx de distinguer les êtres humains des autres animaux et, davantage encore, qu'il conçoit comme étant au fondement de l'historicité de la socialité humaine. Chez les êtres humains, l'attention consciente nécessaire à l'accomplissement d'activités déterminées n'est pas, le plus souvent, entièrement mobilisée dans leur réalisation effective. N'étant pas entièrement submergés par le cours de réalisation de leur activité, ils disposent d'une capacité réflexive qui leur permet de prendre un certain recul à son égard, en posant celle-ci comme objet de leur conscience. Ils sont donc en mesure d'interrompre le cours normal de leur activité afin de la questionner, de la moduler, voire d'élaborer de nouvelles manières de faire en développant de nouvelles techniques ainsi qu'en employant de nouveaux matériaux et instruments. Autrement dit, les êtres humains font preuve de créativité et d'inventivité à l'égard des formes particulières qu'emprunte leur activité, exerçant ainsi une capacité d'autodétermination au cours même de son déploiement. Contrairement aux animaux, ils ne se limitent pas à reproduire des schèmes de comportements relativement similaires en se rapportant à des objets également similaires. Ils peuvent élaborer de nouvelles formes de pratiques tout

10 Ibid., p. 200 ; nous soulignons.

${ }^{11}$ Idem. 
en intégrant de nouveaux objets à l'horizon de leur pratique, voire en étendant celle-ci à l'ensemble de la nature qu'ils aménagent progressivement en fonction des besoins qu'ils déterminent socialement ${ }^{12}$.

En ce sens, les êtres humains constituent des êtres fondamentalement historiques, développant leur pratique, de génération en génération, sous un ensemble de formes distinctes. Les besoins qu'ils éprouvent, les objets dont ils font usage, les pratiques à travers lesquelles ils les produisent et les consomment revêtent tous, pour Marx, un caractère social et historique ${ }^{13}$. La spécificité du métabolisme qu'entretiennent les êtres humains envers la nature emprunte donc la forme d'un double procès à travers lequel ils transforment la nature tout en se transformant eux-mêmes en parallèle. Or, ces considérations d'ordre ontologique qui sont développées par Marx dès les Manuscrits de 1844 se trouvent réitérées dans Le Capital. En effet, juste après avoir défini le travail comme un procès métabolique au fil duquel l'être humain transforme la nature, Marx ajoute qu' « en agissant sur la nature extérieure et en la modifiant par ce mouvement, il modifie aussi sa propre nature ${ }^{14} »$. L'activité humaine est donc comprise comme un double procès à travers lequel les êtres humains s'engendrent eux-mêmes comme sujets sociohistoriques tout en engendrant le monde objectif qu'ils habitent, ils se transforment eux-mêmes tout en transformant la nature.

En somme, l'activité humaine est pour Marx fondamentalement historique et son histoire repose sur un procès dialectique: la réflexivité qui caractérise l'activité humaine s'appuie sur les formes historiquement développées et subjectivement assumées sous lesquelles elle s'incarne historiquement afin d'en élaborer de nouvelles. Ainsi, non seulement conçoit-il les êtres humains comme étant avant tout des êtres agissants, il considère également que cette activité s'incarne toujours, d'une part, sous un ensemble de formes momentanément déterminées, c'est-à-dire sociohistoriques, et constitue toujours, d'autre part, l'occasion de développements ultérieurs, se révélant au cœur de leur historicité. Si nous insistons de la sorte sur la volonté et la conscience comme caractéristiques essentielles de l'activité humaine chez Marx, c'est que les enjeux qui se rattachent à la soumission de la pratique sociale au capital touchent précisément à ces deux aspects, particulièrement en ce qui concerne la soumission virtuelle. En effet, la capacité réflexive qui caractérise l'activité humaine est au cœur de l'emprise que cherche à exercer le capital à son endroit, dont le résultat est la mise en place de nouvelles manières de produire. Dans cet ordre d'idées, le capital participe donc pleinement à l'exploration et au développement des potentialités de l'agir humain. Il participe au double procès à travers lequel les êtres humains s'engendrent tout en engendrant le monde qui les entoure, mais de telle façon qu'il emprunte ici la

12 C'est ce que Marx désigne, dans les Manuscrits de 1844, sous la notion d' « essence générique ». Sur cette question, nous renvoyons à un article antérieur : Éric N. Duhaime, «L'essence générique comme inventivité humaine », Les Cahiers philosophiques de Strasbourg, n 41, p. 99-116.

13 Karl Marx, Manuscrits économico-philosophiques de 1844, op. cit., p. 147.

14 Karl Marx, Le Capital, livre 1, op. cit., p. 199-200. 
forme d'un procès d'aliénation, c'est-à-dire que les formes organisationnelles et techniques qui en résultent se présentent face aux travailleurs sous la forme de puissances étrangères qui les dominent.

\section{La soumission formelle et la valorisation de pratiques sociales préexistantes}

L'avènement du mode de production capitaliste repose pour Marx sur une double condition de possibilité, à savoir le travail salarié et les marchés. Premièrement, le travail salarié découle, en termes philosophiques, de la séparation des êtres humains à l'égard de la nature qui constitue la condition de leur agir et de la satisfaction de leurs besoins ou, en termes sociologiques, de la soustraction et de l'appropriation privée des moyens de production. À son origine, cette séparation est tributaire d'un bouleversement historique majeur des rapports sociaux d'appropriation qui renvoie au mouvement des enclosures de l'Angleterre du XVI ${ }^{\mathrm{e}}$ au XVIII ${ }^{\mathrm{e}}$ siècle. En effet, les enclosures correspondent à une transformation centrale des modalités institutionnelles qui régulaient l'accès aux terres - principal moyen de production de l'époque -, marquant le passage d'un régime fondé sur les rapports de droits et obligations caractéristiques du féodalisme à un régime fondé sur la propriété privée caractéristique du capitalisme15. Deuxièmement, en parallèle à cette transformation, le marché s'imposa comme une médiation institutionnelle centrale pour les acteurs sociaux, et ce, aussi bien pour les travailleurs salariés que pour les capitalistes. Pour les premiers, le marché devint indispensable à la vente de leur force de travail et à l'achat de leurs moyens de subsistance. Pour les seconds, il devint indispensable en ce qui concerne l'accès aux moyens de production et à l'écoulement des biens produits. Toutefois, de manière plus spécifique, c'est l'achat et la vente de la force de travail qui marqua l'avènement du mode de production capitaliste, ce dernier reposant sur le rapport capital-travail, c'està-dire sur la valorisation du capital opérée par l'exploitation du travail d'autrui et l'extraction de survaleur ${ }^{16}$. Or, l'instauration du rapport capital-travail implique que le procès de travail, constituant a priori, comme nous l'avons vu, un double procès au fil duquel les êtres humains transforment la nature tout en se transformant eux-mêmes, fut réduit à l'état de moyen employé par le capital en vue d'opérer son procès d'autovalorisation cumulative :

le travail n'est plus qu'un moyen grâce auquel une somme donnée de valeurs, soit une masse déterminée de travail objectivé, absorbe du travail vivant, en vue de se conserver et de s'accroître. Le procès

\footnotetext{
15 Ellen Meiksins Wood, L'origine du capitalisme. Une étude approfondie, trad. F. Tétreau, Montréal, Lux, 2009, p. 172-174.

${ }^{16}$ Karl Marx, Un chapitre inédit du « Capital », op. cit., p. 188-189.
} 
de travail est donc procès d'auto-valorisation du travail objectivé grâce au travail vivant ${ }^{17}$.

Voilà, au sens le plus général, ce que Marx cherche à problématiser sous le concept de « soumission » du travail au capital.

Cette soumission revêt chez lui une double signification, étant entendue à la fois comme « subsomption » et comme « subordination ». Comme subsomption, d'un côté, dans la mesure où la réduction du procès de travail à l'état de moyen suppose que celuici ne constitue désormais qu'un procès particulier au sein d'un ensemble de procès plus vaste. En effet, ce n'est qu'en étant combiné au procès de circulation que le procès de travail participe au procès de valorisation du capital. C'est pourquoi le mode de production capitaliste peut être compris comme l'unité du procès de circulation et du procès de travail en tant que procès de valorisation. Comme subordination, d'un autre côté, dans la mesure où la réduction du procès de travail à l'état de moyen implique qu'il se déploie désormais sous l'autorité du capitaliste. Puisque les éléments qui entrent dans le procès de travail sont achetés par le capitaliste sur les marchés - comme métamorphose d'une somme d'argent sous forme de capital variable (force de travail) et de capital constant (moyens de production) -, ceux-ci lui appartiennent de plein droit, de telle sorte que le capitaliste acquiert un rôle de direction à l'égard du procès de travail sur la base du seul exercice de son droit de propriété, tandis que les travailleurs se trouvent quant à eux confinés à un rôle d'exécution ${ }^{18}$.

Ainsi, la « soumission formelle » du procès de travail au capital correspond à l'intégration de pratiques sociales préexistantes, encadrées auparavant par d'autres modalités institutionnelles, au mode de production capitaliste, de telle sorte qu'elles se trouvent désormais prises en charge et mobilisées par le capital dans le cadre du procès de valorisation. Ce fut le cas, d'abord, des pratiques agricoles qui étaient auparavant régies par un régime féodal de droits et obligations et, ensuite, des pratiques artisanales qui étaient régies par les guildes artisanales et les corporations de métier. Cette première forme de soumission est qualifiée de «formelle » dans la mesure où elle laisse a priori inchangé le procès de travail lui-même, la contrainte qui s'exerce à son endroit étant extérieure à son déroulement effectif. Quoique le travailleur réalise désormais son métier au sein d'une entreprise privée et sous l'autorité du capitaliste, il demeure néanmoins détenteur du savoir-faire nécessaire à la réalisation de son travail, maîtrisant et surplombant l'ensemble des opérations à accomplir et mobilisant pour ce faire ses propres connaissances et compétences. L'exploitation et la contrainte qui s'exercent envers son travail renvoient à l'extraction de survaleur absolue qui est obtenue par un étirement de la journée de travail. De cette façon, l'usage de la force de

17 Ibid., p. 173.

${ }^{18} \mathrm{Ibid}$., p. 152. Sur cette question et, de façon plus générale, sur la distinction entre soumission formelle et réelle du procès de travail au capital chez Marx, voir Éric Pineault, « Capital, valeur et réversibilité : recherche sur les fondements de l'approche marxienne du capital financier », dans Marx philosophe, dir. Olivier Clain, Québec, Nota bene, 2009, p. 211-259. 
travail dans le procès de production et la quantité de valeur créée au cours de la journée de travail se révèlent supérieurs à la valeur d'échange de la force de travail qui est déterminée quant à elle sur les marchés, remise au travailleur sous forme de salaire et dont la grandeur équivaut à la valeur d'échange d'une somme déterminée de biens de subsistance. Le différentiel entre la valeur d'usage et la valeur d'échange de la force de travail correspond alors à la réalisation d'un « surtravail » qui est accumulé sous forme de survaleur. Pour extraire cette survaleur, le capitaliste s'assure toutefois de tirer le maximum des facteurs de production qu'il achète sur les marchés et qu'il mobilise dans son entreprise, dont l'usage et les fruits lui appartiennent en tant que propriété privée. C'est pourquoi, affirme Marx, le capitaliste met en place un « code pénal » qui vise à éviter le gaspillage de matériaux, l'usure prématurée des instruments ou encore les pertes de temps de travail dues à la fainéantise, ce à quoi se limitent pour le moment le rôle de direction et la réflexivité que le capitaliste exerce à l'égard du procès de travailis.

Bien que la contrainte exercée sur le procès de travail demeure extérieure à son déroulement effectif, dans la mesure où la soumission formelle du procès de travail au capital vise l'extraction de survaleur absolue, il en résulte néanmoins une détermination nouvelle de la signification des cinq moments formels qui en sont constitutifs :

1. L'extraction de survaleur se superpose désormais à l'idée - représentation mentale de l'objet d'usage à produire -, comme finalité générale du procès de travail.

2. Les opérations ne sont maintenant considérées que d'un point de vue purement quantitatif, soit en fonction de la durée temporelle au cours de laquelle il y a une dépense de force de travail qualitativement indifférenciée, à laquelle correspond le concept de « travail abstrait » par opposition au « travail concret ».

3. Les moyens de travail ne sont remis aux travailleurs que dans le but d'assurer le transfert de cette dépense de travail indifférencié à la matière.

4. L'objet ne constitue désormais que le support matériel permettant d'assurer la coagulation de la dépense de travail abstrait.

5. Le résultat ne s'incarne plus simplement dans la matérialisation effective d'un objet utile destiné à un usage social particulier, mais plus spécifiquement dans la production d'une marchandise destinée au marché, qui constitue donc le support d'une valeur d'échange, mais aussi et surtout d'une survaleur ${ }^{20}$.

19 Karl Marx, Le Capital, livre 1, op. cit., p. 208 et 220.

20 Ibid., p. 210 sq. 


\section{La soumission réelle et le bouleversement des procédés de production}

Si la soumission formelle correspond à l'intégration de pratiques sociales préexistantes au mode de production capitaliste et se caractérise par l'exercice d'une contrainte qui demeure extérieure au déroulement effectif du procès de travail lui-même, la soumission réelle correspond quant à elle à la transformation en profondeur, à la fois organisationnelle et technique, des pratiques intégrées au mode de production capitaliste. On assiste alors à une radicalisation de la prise en charge du procès de travail par le capital qui se traduit par l'accentuation de la réflexivité et du rôle de direction qu'exerce le capitaliste à son endroit, et ce, en ciblant des moments spécifiques du procès de travail. Cette transformation du procès de travail qui constitue pour Marx la «tendance spécifique ${ }^{21} \gg$ du mode de production capitaliste repose sur l'extraction de survaleur relative. En comparaison à la survaleur absolue, la survaleur relative renvoie à la réduction de la partie de la journée de travail au cours de laquelle se trouve remboursée la quantité de valeur d'échange reçue par le travailleur sous forme de salaire - le « travail nécessaire »- et à l'augmentation inversement proportionnelle de la partie de la journée où se trouve produite une survaleur - le « surtravail ${ }^{22} »$. Puisque la valeur d'échange de la force de travail correspond à la valeur d'un panier de biens d'usage assurant la subsistance et la reproduction des travailleurs, la réduction de la partie de la journée consacrée au remboursement de cette valeur implique une diminution généralisée de la valeur d'échange des biens qui entrent dans la consommation des travailleurs. Ce résultat est lui-même obtenu par l'augmentation généralisée de la productivité des procédés de production qui en sont à la base, ce qui permet de diminuer la valeur d'échange des marchandises qui en résultent et, par conséquent, la valeur d'échange de la force de travail elle-même.

Or, malgré les bénéfices qui en découlent pour l'ensemble des capitalistes, il ne s'agit pas là, d'après Marx, d'un effet qui serait visé par ceux-ci de façon concertée. Cela dit, il n'en demeure pas moins un avantage majeur incitant les capitalistes singuliers à augmenter la productivité des procédés de production qu'ils emploient dans leurs entreprises respectives. En effet, l'augmentation du rendement du travail permet de produire des marchandises dont la "valeur individuelle» est inférieure à leur " valeur sociale », cette dernière renvoyant à la moyenne déterminée sur les marchés. Puisque la valeur d'échange à laquelle est écoulée une marchandise sur les marchés renvoie à cette moyenne - et non au temps de travail singulier qui a été nécessaire pour la produire -, il en résulte un différentiel qui permet au capitaliste de s'approprier une "survaleur supplémentaire 23 ». Conséquemment, les travailleurs qu'il emploie remboursent plus rapidement la valeur d'échange qu'ils reçoivent sous forme de

\footnotetext{
${ }^{21}$ Karl Marx, Manuscrits de 1857-1858 (« Grundrisse »), t. 2, op. cit., p. 185.

22 Karl Marx, Le Capital, livre 1, op. cit., p. 354.

23 Ibid., p. 357.
} 
salaire, alors que le surtravail qu'ils réalisent s'étire de façon inversement proportionnelle. L'extraction de cette survaleur supplémentaire se maintient jusqu'au moment où, compte tenu de la dynamique concurrentielle des marchés, le nouveau procédé qui a été mis en place par le capitaliste novateur est adopté de manière généralisée par l'ensemble des entreprises concurrentes. À partir de ce moment, la survaleur supplémentaire se trouve réduite à néant alors que la valeur individuelle des marchandises produites au moyen du nouveau procédé s'impose désormais comme nouvelle moyenne et donc comme nouvelle valeur sociale.

C'est pourquoi, affirme Marx, le capitaliste cherche à tirer le maximum de profits de son nouveau procédé de production avant d'être imité par la concurrence, en faisant fonctionner ses nouvelles machines à plein régime, de jour comme de nuit. Il se trouve alors emporté par une sorte de fièvre qui est impulsée par l'appât de gains faramineux que permet de générer l'extraction de la survaleur supplémentaire. Or, voilà précisément ce que Marx compare à « cette première saison d'amour » (erste Zeit der jungen Liebe), autrement dit à la période de «lune de miel» et à la passion intense qui s'empare des amoureux lors des premiers moments d'un amour naissant :

Pendant cette période de transition, où l'emploi des machines reste une sorte de monopole, les gains sont donc extraordinaires, et le capitaliste cherche à exploiter le plus radicalement possible « cette première saison d'amour » par la plus grande prolongation possible de la journée de travail. L'importance du gain aiguise sa fringale de gains plus grands encore 24 .

Cette fièvre momentanée qui emporte le capitaliste se résorbe toutefois lorsque son nouveau procédé de production se trouve imité par la concurrence et que celui-ci s'impose désormais comme nouvelle manière socialement déterminée de produire un bien d'usage particulier.

Cela dit, l'effet combiné des innovations éparses et de la dynamique concurrentielle des marchés, en augmentant la productivité et en abaissant de proche en proche la valeur d'échange des marchandises qui entrent dans la consommation des travailleurs, permet de diminuer la valeur d'échange de leur force de travail25. Dans cet ordre d'idées, la soumission réelle et l'extraction de survaleur relative impliquent une transformation progressive du procès de travail, dont Marx a cherché à problématiser le développement sous trois grandes phases historiques. Comme nous le verrons, ce développement s'est par ailleurs accompagné d'un double procès de déqualification des travailleurs et de requalification d'une minorité d'entre eux, et dont l'objet de travail correspondra désormais au procès de travail lui-même.

24 Karl Marx, Le Capital, livre 1, op. cit., p. 456.

25 Sur tout ce développement, voir ibid., p. 354-359. 
La première de ces phases, la « coopération simple », renvoie au regroupement d'un ensemble de travailleurs dans un même établissement, sous l'autorité du capitaliste. Elle se caractérise ainsi par la massification du procès de travail. Dans ce contexte, les travailleurs réalisent chacun de leur côté l'ensemble des opérations nécessaires à la production d'une marchandise, les unes à la suite des autres, en demeurant ainsi détenteurs du savoir-faire nécessaire à l'accomplissement du travail et en surplombant l'ensemble des opérations à effectuer. L'augmentation de la productivité résulte de deux facteurs qui réfèrent aux conditions subjectives et objectives du procès de travail. Du côté des conditions subjectives, soit de l'activité réalisée par les travailleurs, l'effort combiné des travailleurs leur permet de réaliser certaines tâches dont ils ne sauraient s'acquitter individuellement avec autant d'aisance ou de rapidité : par exemple, en formant une chaîne humaine pour charger ou décharger un camion ou encore en se mettant à plusieurs pour soulever et déplacer un objet d'un poids important. De même, le regroupement des travailleurs entraîne selon Marx un effet d'émulation où chacun tend à apprendre et cherche à dépasser les performances des autres. Du côté des conditions objectives, soit des moyens de production, le rassemblement d'un ensemble de travailleurs dans un même établissement génère des économies d'échelle en lien aux infrastructures nécessaires et aux outils que se partagent désormais les travailleurs ${ }^{26}$. Quant à son impact sur le procès de travail, la massification du procès de travail suppose une organisation de plus en plus systématique de la fonction de direction qui devient elle-même, affirme Marx, une " condition de la production 27 ". Le rôle de direction, dont le capitaliste se réserve les plus hautes fonctions, se trouve alors réfracté sous un ensemble de sous-fonctions structurées de manière hiérarchique, de telle sorte que l'objet de travail d'un ensemble de travailleurs consiste désormais à surveiller le travail des autres ${ }^{28}$.

La seconde phase, la «manufacture », repose sur la décomposition et la recomposition des tâches nécessaires à la production d'une marchandise déterminée qui se trouvent réparties entre un ensemble de travailleurs distincts qui ne réalisent désormais qu'une tâche spécialisée à répétition. Cette seconde phase se caractérise ainsi par la division des opérations du procès de travail ou ce que Marx appelle la « division de détail » - par opposition à la « division sociale» du travail qui renvoie à la spécialisation sociale par métiers 29 . Au cours de cette phase, l'augmentation de la productivité cible avant tout les conditions subjectives du procès de travail et, plus spécifiquement encore, le moment formel que constituent les opérations comme ensemble de manipulations effectives visant à mettre en forme la matière. Conformément au célèbre exemple de la manufacture d'épingles d'Adam Smith, dont Marx s'inspire ici directe-

${ }^{26}$ Ibid., p. 365-367.

27 Ibid., p. 372.

28 Ibid., p. 374. Sur cette question de l'organisation et de la prise en charge du travail d'autrui, voir François L'Italien, Béhémoth Capital. Genèse, développement et financiarisation de la grande corporation, Montréal, Nota bene, 2016, p. 37-50.

29 Karl Marx, Le Capital, livre 1, op. cit., p. 395. 
ment, le confinement des travailleurs à des tâches spécialisées favorise l'accroissement de leur habileté pour la réalisation de tâches uniques et répétitives, l'économie de pertes de temps relatives au passage d'une tâche à une autre ainsi que l'accroissement de l'efficacité des outils qui font l'objet d'améliorations continues en fonction d'usages spécifiques ${ }^{30}$. Eu égard au procès de travail, cette spécialisation des tâches suppose toutefois une déqualification progressive des travailleurs dont la formation se limite désormais à l'apprentissage d'une tâche exclusive et répétitive. Ainsi, la manufacture constitue un point de rupture à l'égard du savoir-faire artisanal auquel elle s'oppose de manière frontale, allant jusqu'à faire "une spécialité du manque même de tout développement ${ }^{11}$ ». Cela dit, la division des tâches suppose l'avènement d'une nouvelle forme de travail qualifié dont l'objet porte alors sur l'organisation du travail lui-même. Celle-ci consiste dans la répartition structurée des opérations décomposées du procès de travail au fil d'une chaîne de travailleurs qui sont proportionnellement distribués en sous-groupes spécialisés et en fonction du temps nécessaire à l'accomplissement de chacune des opérations. Le procès de travail est donc à la fois décomposé en de multiples opérations spécialisées et recomposé sous la forme d'une chaîne de travailleurs répartis de façon proportionnelle, de telle sorte qu'il puisse se dérouler de manière fluide et ininterrompue. En ce sens, la position de surplomb qui conférait une unité synthétique au procès de travail et qui reposait sur le savoir-faire de l'artisan se trouve désormais accaparée par ces travailleurs qualifiés qui organisent désormais le travail des autres, l'ensemble des travailleurs spécialisés ne constituant alors que les rouages partiels d'un procès d'ensemble dont l'unité repose sur l'organisation de leur effort combiné.

La troisième phase, la « grande industrie », repose sur le recours de plus en plus systématique à la machinerie à travers l'application des découvertes de la science et de la technologie aux processus industriels. Elle se caractérise ainsi par l'automatisation du procès de travail. Au cours de cette phase, l'augmentation de la productivité cible avant tout les conditions objectives du procès de travail et, plus spécifiquement encore, le moment formel que constitue le moyen de travail. L'utilisation de machines de plus en plus intégrées qui empruntent la forme d'une chaîne de production automatisée et qui comportent une source d'énergie autonome avec l'usage de la vapeur permet d'affranchir le procès de travail des limitations corporelles des travailleurs ${ }^{32}$. Dès lors, la quantité d'opérations réalisées simultanément par les machines-outils, la cadence et la puissance des procédés de production s'en trouvent radicalement augmentées. Eu égard au procès de travail, il en résulte un transfert des opérations désormais prises en charge par les machines alors que l'activité des travailleurs se limite de plus en plus à surveiller le bon fonctionnement de celles-ci et à intervenir ponctuellement lors de défaillances ou de dysfonctionnements. Pour Marx, la grande industrie sonne ainsi le

\footnotetext{
30 Ibid., p. 381-384.

31 Ibid., p. 394.

32 Ibid., p. 420.
} 
glas du savoir-faire artisanal dont la destruction fut amorcée avec la manufacture33. Cela dit, l'automatisation du procès de travail suppose l'avènement d'une nouvelle forme de travail qualifié, celui des mécaniciens et des ingénieurs dont l'objet de travail porte précisément sur les moyens de production. Ce travail consiste alors à concevoir, entretenir et réparer les machines. En ce sens, la position de surplomb qui confère une unité au procès de travail se trouve désormais prise en charge par ces travailleurs qualifiés. Le caractère synthétique du procès de travail s'incarne maintenant dans l'organisation systématique des machines intégrées qui réalisent, les unes à la suite des autres, l'ensemble des opérations nécessaires à la production de marchandises déterminées.

Pour Marx, la soumission réelle du procès de travail au capital et l'augmentation de la productivité qui s'y rattache assurent un développement des "potentialités » du procès de travail. Toutefois, ce développement, à la fois organisationnel et technique, s'opère au moyen d'une prise en charge de la réflexivité caractéristique de l'activité humaine par le capital et ses représentants, et donc sur la base du droit qu'exerce le capitaliste à l'égard de sa propriété privée et dans le but d'extraire une survaleur relative. Ce développement se trouve ainsi entièrement assujetti à la finalité qui est propre au mode de production capitaliste - la valorisation du capital -, empruntant alors la forme d'un procès d'aliénation compris comme un devenir étranger des potentialités de l'activité humaine34. En effet, les capacités organisationnelles et techniques du procès de travail qui se trouvent développées se consolident face aux travailleurs sous la forme de puissances étrangères, comme modes d'existence du capital lui-même :

avec le développement du mode de production spécifiquement capitaliste, ce ne sont plus seulement les objets - ces produits du travail, en tant que valeurs d'usage et valeurs d'échange - qui, face à l'ouvrier, se dressent sur leurs pieds comme «capital », mais encore les formes sociales du travail qui se présentent comme formes de développement du capital, si bien que les forces productives, ainsi développées, du travail social apparaissent comme forces productives du capital: en tant que telles, elles sont "capitalisées », en face du travail. En fait, l'unité collective se trouve dans la coopération, l'association, la division du travail, l'utilisation des forces naturelles, des sciences et des produits du travail sous forme des machines. Tout cela s'oppose à l'ouvrier individuel comme quelque chose qui lui est étranger et existe au préalable sous forme matérielle; qui plus est, il lui semble qu'il

\footnotetext{
33 Ibid., p. 415.

34 Sur le concept d'aliénation chez Marx compris comme un « devenir étranger », voir Franck Fischbach, «Présentation » dans Karl Marx, Manuscrits économico-philosophiques de 1844, op. cit., p. 7-71.
} 
n'y ait contribué en rien, ou même que tout cela existe en dépit de ce qu'il fait35.

En parallèle, tout savoir-faire est progressivement éliminé des activités accomplies par les travailleurs qui se retrouvent selon Marx réduits à l'impuissance. Non seulement sont-ils séparés des conditions qui leur permettraient de réaliser leur activité de façon autonome, ils ne peuvent plus désormais réaliser un travail et satisfaire leurs besoins que dans un contexte qui n'exige d'eux que la réalisation d'une activité réduite à sa plus simple expression, limitée à la surveillance du bon fonctionnement des machines : "la machine ne libère pas l'ouvrier du travail, mais ôte au travail son contenu ${ }^{36}$ ». Même en ce qui concerne la minorité de travailleurs qualifiés dont l'objet de travail se rattache désormais à la surveillance, à l'organisation et à l'amélioration technique des procédés de production, le résultat de leurs activités se consolide pour Marx sous la forme de puissances étrangères qui appartiennent au capitaliste, en participant, de concert avec les formes de travail déqualifié qu'ils prennent pour objet, à la valorisation du capital. Or, c'est précisément dans le prolongement de ce double procès de déqualification des travailleurs et de requalification d'une minorité d'entre eux que se comprend la soumission virtuelle de la pratique sociale au capital.

\section{La soumission virtuelle et la détermination de l'avenir}

Si la soumission formelle correspond à l'intégration de pratiques sociales préexistantes au mode de production capitaliste, et la soumission réelle à la transformation organisationnelle et technique des pratiques ainsi intégrées, la soumission virtuelle correspond quant à elle à la détermination des pratiques sociales futures. Elle porte en effet sur le contrôle des possibilités et des potentialités de l'activité humaine, ce qui se traduit notamment par la détermination de nouveaux objets d'usage et de nouveaux besoins, de même que par la détermination des nouvelles pratiques de production et de consommation qui s'y rattachent. En ce sens, la soumission virtuelle de la pratique sociale au capital porte sur la spécificité caractéristique de l'activité humaine en tant qu'activité consciente, la distance réflexive qui lui est propre étant elle-même tributaire de la virtualité qu'elle acquiert comme activité comportant une dimension irréductiblement idéelle et symbolique.

En tant que système de symboles unifiés, le langage rend possible la prise de parole qui permet aux êtres humains non seulement d'exprimer et de partager leurs expériences singulières dans le cadre de rapports intersubjectifs, mais également de désigner et de discuter des objets de leur pratique et de leurs pratiques elles-mêmes en dehors de la présence effective des premiers ou de la réalisation effective des secondes. Ainsi, à la différence des animaux pour lesquels la discrimination significative d'un

35 Karl Marx, Un chapitre inédit du « Capital », op. cit., p. 250.

$3^{6}$ Karl Marx, Le Capital, livre 1, op. cit., p. 474. 
objet sur le fond que constitue l'environnement se rattache toujours à l'actualisation d'un schème de comportement déterminé, l'activité consciente qui caractérise les êtres humains repose sur la virtualité qu'elle acquiert comme activité médiatisée par le symbolique, ce qui favorise et accroît chez eux la possibilité d'une prise de distance réflexive à l'égard des formes de leurs pratiques et des objets auxquels elles se rapportent. C'est là le cœur de la théorie du symbolique développée par Michel Freitag, qui constitue l'une des dimensions centrales de la vie humaine en comparaison à la vie animale. En effet, si l'activité animale se caractérise par son «actualité », l'activité humaine se caractérise quant à elle par sa "virtualité ». Autrement dit, il existe chez les animaux un rapport de proximité spatiale et temporelle entre les objets qu'ils discriminent au sein de leur environnement et la réalisation de schèmes de comportements particuliers - par exemple le repérage de la proie et le comportement de prédation -, tandis que le langage permet aux êtres humains de discuter des différents objets dont se compose leur monde et de la multiplicité des formes de leur agir sans que ceux-ci ne soient immédiatement présents ou que celles-ci ne soient en cours de réalisation. Or, c'est précisément cette virtualité propre à l'activité humaine, en tant qu'elle est médiatisée symboliquement, qui est au fondement de la réflexivité des êtres humains et au cœur de leur historicité, c'est-à-dire du double procès au fil duquel les êtres humains s'engendrent comme sujets sociohistoriques tout en engendrant le monde objectif qu'ils habitent 37 .

Bien que Marx lui-même n'ait pas élaboré une théorie complète et systématique du symbolique comme aspect fondamental de la vie humaine - en abordant la question du langage de façon momentanée et partielle, et en limitant le plus souvent celui-ci à une dimension relativement instrumentale, c'est-à-dire comme simple moyen de communication-, cette virtualité s'observe néanmoins chez lui dans le moment formel du procès de travail que constitue l'idée. En effet, puisqu'elle est présentée comme une représentation mentale et a priori de l'objet que l'on cherche à produire, des propriétés formelles et matérielles qu'il doit comporter en fonction de l'usage social auquel il est destiné, l'idée relève de l'anticipation et donc de la virtualisation, au sens où l'entend Freitag. C'est la raison pour laquelle nous proposons le concept de soumission virtuelle de la pratique sociale au capital afin de désigner l'emprise que le capital exerce à l'égard des potentialités et du devenir des formes de la pratique humaine, soit comme production de nouvelles idées et donc comme développement de nouveaux objets d'usage et de nouveaux besoins auxquels se rattachent toujours de nouvelles pratiques de production et de consommation.

Au-delà du capitalisme industriel qui a caractérisé l'époque de Marx et qui a retenu son attention, la soumission virtuelle de la pratique sociale au capital se rattache à l'avènement du capitalisme avancé qui s'est mis en place au tournant du $\mathrm{XX}^{\mathrm{e}}$ siècle.

37 Michel Freitag, « Actualité de l'animal, virtualité de l'homme », Conjonctures, n ${ }^{\text {os }} 33-34,2001-2002$, p. 99-154; Dialectique et société, vol. 2 : Introduction à une théorie générale du symbolique, Montréal, Liber, 2011, p. 152, 171, 198-199, 246 et 319. 
À l'encontre du capitalisme industriel du $\mathrm{XIX}^{\mathrm{e}}$ siècle, le capitalisme avancé se caractérise par l'importance grandissante de la grande corporation de droit privé comme sujet central du mode de production capitaliste ainsi que par les stratégies de contrôle d'ordre monopolistique qu'elle privilégie à l'égard des marchés. L'apparition de la grande corporation s'est en effet accompagnée d'une transformation importante de la dynamique des marchés, la forme corporative que constitue le holding favorisant les exercices de fusions et d'acquisitions horizontales - comme monopolisation d'une branche de production donnée - et verticales - comme intégration de l'ensemble des étapes nécessaires à la production d'un bien fini. La multiplication des exercices de fusions et d'acquisitions a pour conséquence une internalisation progressive des marchés, les échanges entre les unités de production s'opérant désormais à l'intérieur même des corporations, entre leurs différentes filiales, et non plus entre des entreprises indépendantes par la médiation des marchés 38 .

Or, cette internalisation des échanges a pour corollaire une transformation des relations externes que les corporations entretiennent entre elles sur les marchés, ces derniers empruntant une forme de plus en plus oligopolistique. La concurrence par les prix qui était caractéristique du capitalisme industriel cède alors la place à de nouvelles stratégies concurrentielles visant à éviter les guerres de prix. D'un côté, les corporations en viennent à privilégier des pratiques de co-respect en ce qui concerne la détermination des prix de leurs marchandises sur les marchés39. De l'autre, comme le soutient Veblen, elles misent sur l'acquisition d' «avantages différentiels » qui constituent désormais les nouvelles armes de la concurrence, à savoir : le contrôle de l'approvisionnement en matières premières, de procédés de production ou d'inventions au moyen de brevets ou encore le contrôle de la consommation à travers le marketing et la publicité40. Pour Veblen, la détention d'avantages différentiels permet d'opérer des perturbations sur les marchés, c'est-à-dire qu'ils confèrent aux corporations une capacité à déterminer les prix de leurs marchandises en assurant un contrôle sur les volumes de production et de mise en circulation 41 .

Dans cette perspective, les revenus générés par les corporations ne se fondent plus seulement sur le contrôle interne qu'elles exercent à l'égard des procédés de production, mais également sur le contrôle externe qu'elles acquièrent vis-à-vis des marchés. Autrement dit, leurs revenus ne reposent plus seulement sur la mobilisation d'un capital productif dans le cadre du procès de production, mais également sur la mobilisation d'avantages différentiels dans le cadre du procès de circulation. Dans ce contexte, la soumission virtuelle correspond à l'extraction d'une nouvelle forme de

\footnotetext{
38 Michel Freitag, L'impasse de la globalisation: une histoire sociologique et philosophique du capitalisme, Montréal, Écosociété, 2008, p. 167-169.

39 Paul A. Baran et Paul M. Sweezy, Le capitalisme monopoliste, trad. C. Passadéos, Paris, Maspero, 1979, p. 66-72.

40 Thorstein Veblen, The Theory of business enterprise, Clifton, Kelley, 1973, p. 55 et 138.

${ }^{41}$ Ibid., p. 25-35.
} 
survaleur que l'on peut qualifier, en nous inspirant du concept de Veblen, de survaleur différentielle, et qui implique l'arrimage de l'exploitation exercée à l'égard des travailleurs dans la production à la position de force exercée envers les consommateurs sur les marchés. Cette survaleur différentielle se comprend à partir de la « survaleur supplémentaire » que nous avons abordée en discutant de la survaleur relative et qui est générée par l'introduction de nouveaux procédés de production. Comme nous l'avons vu, l'augmentation de la productivité se traduit par la production de marchandises à une valeur individuelle moindre que leur valeur sociale. Et, puisque la valeur d'échange à laquelle elles peuvent être écoulées correspond à leur valeur sociale moyenne et non à leur valeur individuelle, il en résulte l'extraction d'une survaleur supplémentaire qui perdure jusqu'au moment où le nouveau procédé se généralise par effet de concurrence, de telle sorte que la valeur individuelle s'impose alors comme nouvelle moyenne sociale et que la valeur supplémentaire se trouve réduite à néant.

Or, les avantages différentiels que détiennent les corporations visent justement à neutraliser cet effet qu'opère la concurrence, ce qui leur confère alors une capacité à déterminer librement le prix de leurs marchandises sur les marchés. Elles parviennent de la sorte à maintenir de façon artificielle la valeur sociale de leurs marchandises audelà de leur valeur individuelle, d'où l'extraction indéfinie d'une survaleur supplémentaire qui perdure aussi longtemps qu'est maintenu l'avantage différentiel. Pour reprendre l'image de Marx, la fièvre qui emporte le capitaliste disposant du monopole temporaire sur un nouveau procédé de production se prolonge alors sous la forme d'une éternelle saison des amours. Étant donné l'extraction indéfinie de cette survaleur supplémentaire, les travailleurs employés par les corporations remboursent plus rapidement la valeur d'échange qu'ils reçoivent sous forme de salaire, alors que le surtravail qu'ils réalisent s'accroît de façon inversement proportionnelle. Cela dit, puisque les marchandises produites par les corporations sont maintenues à une valeur d'échange artificiellement surélevée, il en résulte une double pression exercée sur les consommateurs, aussi bien sur les travailleurs que sur les entreprises. D'un côté, cette survaleur différentielle implique une pression exercée sur le pouvoir d'achat des travailleurs qui se traduit par la mobilisation et la captation d'une partie surélevée de la valeur d'échange qu'ils consacrent aux biens de subsistance produits par les corporations détenant des avantages différentiels, et ce, au détriment de la valeur d'échange qu'ils peuvent consacrer aux autres biens d'usage qui leur sont nécessaires et des entreprises qui les produisent. De l'autre, elle implique également une pression exercée sur le pouvoir d'achat des entreprises qui se traduit par la mobilisation et la captation d'une partie surélevée de la valeur d'échange qu'elles consacrent à l'acquisition des moyens de production fabriqués par les corporations détenant des avantages différentiels, et ce, au détriment de la survaleur qu'elles parviennent elles-mêmes à générer et qui se trouve partiellement transférée et accaparée par les corporations. Sur la base de cette double pression, il en résulte alors la formation d'une hiérarchie d'entreprises qui repose sur leurs puissances organisationnelles respectives et sur la détention d'avantages différentiels plus ou moins névralgiques, ce qui se traduit par une capacité 
différenciée à extraire et à accaparer une plus ou moins grande partie de la survaleur produite de façon générale dans la société 42 .

Partant de l'analyse sociohistorique et idéaltypique élaborée par Neil Fligstein au sujet des différentes formes d'organisations et de stratégies de contrôle successivement mises en place par les corporations, il est possible de rendre compte du développement historique de la soumission virtuelle sous deux grandes phases historiques distinctes. Tout comme pour les phases de la soumission réelle, ces deux phases de la soumission virtuelle ciblent des moments spécifiques du procès de travail qui font l'objet d'une prise en charge réflexive dans le cadre des stratégies monopolistiques adoptées par les corporations. Faisant suite à la stratégie de contrôle direct qui fut d'abord privilégiée dans le cadre du Great Merger Movement du tournant du XX $\mathrm{XX}^{\mathrm{e}}$ siècle et qui repose sur la stricte monopolisation d'une branche de production par l'entremise de fusions et d'acquisitions horizontales 43 , la première phase de la soumission virtuelle repose sur la stratégie du «contrôle manufacturier » qui s'est progressivement développée et généralisée de 1900 à 1920. Si la stratégie de contrôle direct permet d'assurer un contrôle sur les volumes de production et de mise en circulation des marchandises afin d'en déterminer les prix, elle ne permet toutefois pas d'éviter le surgissement subséquent de nouveaux compétiteurs. Ainsi, le contrôle manufacturier vise quant à lui le renforcement des barrières à l'entrée pour une branche de production déterminée qui décourage l'arrivée de nouveaux compétiteurs. Réalisée au moyen de fusions et d'acquisitions verticales, cette stratégie consiste à intégrer l'ensemble des étapes nécessaires à la production d'un bien d'usage fini au sein d'une même corporation, soit de l'extraction des matières premières à la commercialisation, en passant par les diverses étapes de production44. Cette stratégie a avant tout été développée par des corporations dont les marchandises se rattachaient à l'exploitation de ressources naturelles et qui nécessitaient de faibles transformations, mais de lourds investissements en infrastructures : pâtes et papiers, métallurgie, pétrochimie, etc. Dans le cadre de cette stratégie, l'accès privilégié aux matières premières constitue l'élément stratégique, l'appropriation privée ou l'établissement d'ententes exclusives quant à l'accès aux ressources naturelles permettant de prévenir le surgissement d'éventuels compétiteurs. En ce sens, cette stratégie de contrôle cible le moment formel du procès de travail que constitue l'objet, soit la matière mise en forme au cours du procès de travail. Le contrôle en amont qui est exercé sur l'approvisionnement en matières premières assure donc un contrôle en aval exercé sur les marchés. Il permet de déterminer les volumes de production et de mises en circulation des marchandises, constituant ainsi un avantage différentiel qui prévient le surgissement de nouveaux compétiteurs et confère à son détenteur une capacité à déterminer le prix de ses marchandises sur les marchés.

42 Paul M. Sweezy, The Theory of capitalist development, Londres, Dobson, 1962, p. 274.

43 Neil Fligstein, The Transformation of corporate control, Cambridge, Harvard University Press, 1990, p. 60.

44 Ibid., p. 75. 
La seconde phase de la soumission virtuelle repose sur la stratégie de « contrôle par les ventes et le marketing » qui s'est développée et généralisée de 1920 à 1950. Si la stratégie de contrôle manufacturier permet de décourager d'éventuels compétiteurs, elle se révèle toutefois problématique lors des épisodes de récessions ou de crises économiques. Puisque la réponse consiste à diminuer les volumes de production pour maintenir les prix élevés, elle a pour effet d'envenimer la situation. En comparaison, le contrôle par les ventes et le marketing mise plutôt sur le maintien et la stimulation de la demande en cherchant à fidéliser et à élargir la clientèle45. En ce sens, cette stratégie combine la diversification et la différenciation ${ }^{46}$. D'un côté, la diversification consiste à offrir une gamme de produits connexes comportant des échelons de qualités et de prix variés dans le but de rejoindre la plus grande clientèle possible. De l'autre, la différenciation consiste à promouvoir l'image de marque afin de fidéliser la clientèle et de séduire celle des autres, l'idéal consistant à rendre ses propres marchandises non substituables par celles des compétiteurs. Cette stratégie a été développée avant tout par des corporations dont les marchandises étaient destinées à la consommation domestique : automobiles, électroménagers, produits ménagers, alimentation, etc.

Reposant sur la diversification et la différenciation, cette stratégie cible aussi bien le moment formel du procès de travail que constitue l'idée que celui que constitue le résultat. Premièrement, l'idée consiste, comme nous l'avons vu, dans la représentation mentale des propriétés formelles et matérielles que doit comporter un objet en fonction de l'usage social auquel il est destiné. Or, la diversification vise le contrôle des nouveaux objets d'usage créés socialement, des inventions, et cela au moyen des brevets qui, comme monopoles temporaires reconnus légalement, permettent l'appropriation effective de ces objets d'usage inédits non pas sous une forme concrète et matérielle, mais bien sous une forme générique et immatérielle, soit précisément en tant qu'idées. En ce sens, la diversification est réalisée au moyen de fusions et d'acquisitions stratégiques ainsi qu'à travers la mise sur pied de laboratoires de recherche privés qui visent la commercialisation d'inventions brevetées. Deuxièmement, le résultat consiste, comme nous l'avons vu, dans la matérialisation effective d'un objet concret destiné à répondre à un besoin social déterminé. Or, la différenciation vise le contrôle des marchés à travers la promotion de l'image de marque d'une compagnie et des biens qu'elle produit. En ce sens, la différenciation implique de créer des besoins sociaux pour des objets d'usage inédits, des inventions brevetées, au moyen de campagnes publicitaires. Autrement dit, la stratégie de contrôle par les ventes et le marketing implique aussi bien de développer et de commercialiser de nouveaux objets d'usage que de créer et de stimuler une demande pour ceux-ci. De fait, les objectifs des fusions et des acquisitions stratégiques ou de recherche et développement tendent

45 Ibid., p. 117-118.

46 Ibid., p. 118 et 123 . 
à être déterminés et subordonnés aux objectifs établis par les départements de ventes et de marketing 47 .

La soumission virtuelle de la pratique sociale au capital correspond à la mise en place et à la généralisation de cette nouvelle stratégie de contrôle qui repose sur la mobilisation et la prise en charge réflexive des moments formels du procès de travail que constituent l'idée et le résultat. Elle implique la détermination de nouveaux objets d'usage et de nouveaux besoins auxquels se rattachent de nouvelles pratiques de production et de consommation. Son avènement a toutefois nécessité l'intégration progressive de la production scientifique et technologique à l'intérieur même de la dynamique propre au capitalisme, ce qui a été opéré à travers le développement de la profession d'ingénieur comme apparition d'une nouvelle forme de travail qualifié. De fait, cette profession a explosé en parallèle au développement des grandes corporations et à la nouvelle stratégie d'affaires qui se mettait en place. Au tournant du $\mathrm{XX}^{\mathrm{e}}$ siècle, aux États-Unis, le nombre de laboratoires privés de recherche et développement passait de 300 à 2200 de 1920 à 1940, alors que le pourcentage de brevets détenus par de grandes corporations augmentait de 12 à $75 \%$ de 1885 à 1930 et que le nombre d'ingénieurs passait de 45000 à 230000 de 1900 à $19304^{4}$. Cela dit, la profession d'ingénieur ne s'est pas développée seulement d'un point de vue quantitatif, mais aussi qualitatif, c'est-à-dire sous la forme d'une spécialisation fonctionnelle à la fois verticale et horizontale. En ce sens, le développement de cette profession peut être compris comme le résultat de la soumission formelle et réelle de la production scientifique et technologique au mode de production capitaliste, ce qui entraîna toutefois, en retour, la mise en place de la soumission virtuelle de la pratique sociale au capital.

En premier lieu, la soumission formelle de la production scientifique et technologique au capital correspond à la façon dont Marx problématise lui-même la question en rapport au machinisme qui caractérise la grande industrie de son époque. En effet, le machinisme est au départ développé par des mécaniciens qui sont issus des artisans dont la main-d'œuvre est rendue disponible avec le processus de déqualification des travailleurs qui accompagne la division des tâches caractéristique de la manufacture. Ces mécaniciens puisent alors aux connaissances artisanales ainsi qu'aux découvertes de la science qu'ils cherchent à traduire sous forme d'applications industrielles.

En second lieu, la soumission réelle de cette pratique au capital correspond au développement du métier d'ingénieur qui se consolide avec l'avènement d'écoles à vocation technique et l'adoption de programmes techniques par les grandes universités. En effet, l'institutionnalisation de la formation en ingénierie, opérée à la demande des dirigeants des grandes corporations au tournant du $\mathrm{XX}^{\mathrm{e}}$ siècle, implique une progressive spécialisation fonctionnelle à la fois verticale et horizontale du métier

47 Ibid., p. 127.

${ }^{48}$ Harry Braverman, Travail et capitalisme monopoliste, trad. D. Letellier et S. Niémetz, Paris, Maspero, 1976, p. 139-140 ; David Noble, America by design, Nairobi, Oxford University Press, 1977, p. 39 et 87. 
d'ingénieur49. D'un côté, le développement de programmes de management entraîne une spécialisation verticale. Puisque, au départ, les postes de direction dans les grandes corporations étaient principalement occupés par des ingénieurs - après une quinzaine d'années de pratique en moyenne -, ceux-ci ont incité les écoles techniques et les universités à mettre au point des programmes de management afin de pallier le décalage qu'ils percevaient entre leur formation technique et les fonctions de direction qu'ils occupaient. Or, la mise sur pied de programmes en management a eu pour effet, en contrepartie, de confiner les ingénieurs à des rôles désormais exclusivement techniques. D'un autre côté, le métier d'ingénieur s'est également spécialisé de manière horizontale sous un ensemble de sous-fonctions que l'on peut d'ailleurs rapporter aux différents moments formels du procès de travail chez Marx: les ingénieurs en recherche et développement (idée), les gestionnaires du personnel (opérations), les ingénieurs mécaniques (moyens), les ingénieurs en matériaux (objet) et les spécialistes en marketing et en publicité (résultat). En somme, c'est donc par le développement de la profession d'ingénieur, opéré en parallèle au développement des grandes corporations et à la demande expresse de leurs dirigeants, que la production scientifique et technologique a été intégrée au mode de production capitaliste.

\section{Développement extensif et intensif du mode de production capitaliste}

Eu égard à la soumission formelle et réelle, l'avènement de la soumission virtuelle se comprend dans le cadre d'un développement dialectique. En effet, les rapports qu'entretiennent entre elles ces différentes formes de soumission de la pratique sociale au capital ne doivent pas être compris simplement comme des phases historiques successives, distinctes et mutuellement exclusives, mais plutôt comme étant enchevêtrées de façon cumulative les unes aux autres. D'un côté, la soumission formelle constitue certes la condition de possibilité de la soumission réelle, tout comme cette dernière constitue la condition de possibilité de la soumission virtuelle. Cela dit, d'un autre côté, l'avènement de la soumission virtuelle n'implique pas que la soumission réelle cesse de produire ses effets, pas plus que la soumission réelle n'implique que la soumission formelle cesse de produire les siens. Chez Marx lui-même, comme il le montre dans les Manuscrits de 1857-1858, aussi désignés sous le nom de Grundrisse, la relation de la soumission formelle à la soumission réelle relève d'un rapport dialectique dans la mesure où le développement de l'une entraîne toujours la possibilité d'un développement pour l'autre, et inversement.

C'est pourquoi le développement expansif du capital, sa « reproduction élargie », ne revêt pas seulement une dimension quantitative chez Marx - plus de travailleurs salariés et plus de moyens de production -, mais également qualitative. Elle suppose aussi bien l'intégration progressive de nouvelles formes de pratiques sociales que leur

49 David Noble, America by design, op. cit., p. 33-49. 
bouleversement organisationnel et technique. Dans cette perspective, le caractère expansif du capital en tant que procès de valorisation cumulatif se trouve dépeint comme un développement à la fois extensif et intensif, la soumission formelle et réelle produisant leurs effets par alternance. Si la soumission formelle renvoie à l'intégration d'une pratique sociale préexistante au mode de production capitaliste, celle-ci correspond alors au développement extensif de la logique du capital à une nouvelle sphère de la pratique sociale qui se trouve alors constituée sous la forme d'une nouvelle branche de la production capitaliste. Toutefois, à partir du moment où cette forme de pratique sociale se trouve intégrée au mode de production capitaliste, la soumission réelle commence alors à produire ses effets, si bien que la transformation organisationnelle et technique de cette pratique correspond au développement intensif de cette nouvelle branche de la production. Or, le temps de travail qui se trouve libéré dans cette branche à travers l'intensification des procédés de production permet en retour une nouvelle extension de la logique du capital à une nouvelle sphère de la pratique sociale, et ainsi de suite.

En effet, afin de maintenir et poursuivre son procès de valorisation, le capital qui est accumulé doit continuellement trouver de nouveaux débouchés alors que les branches de production dans lesquelles il est investi jusque-là se retrouvent progressivement saturées. Et, en investissant de nouvelles sphères de la pratique sociale qu'il constitue sous la forme de nouvelles branches de production, il peut alors mobiliser la main-d'œuvre rendue disponible par l'intensification de la production dans les autres branches de production:

La tendance du capital est, bien sûr, de lier la survaleur absolue à la survaleur relative ; donc allongement maximum de la journée de travail avec le nombre maximum de journées de travail simultanées, allant de pair avec la réduction au minimum, d'une part, du temps de travail nécessaire, d'autre part, du nombre nécessaire de travailleurs [...]. Une conséquence nécessaire en est la plus grande multiplication possible de la valeur d'usage du travail - ou encore des branches de production - si bien que la production du capital accélère d'un côté de façon permanente et nécessaire le développement de l'intensité de la force productive du travail, tout comme, de l'autre côté, elle engendre la variété illimitée des branches du travail, c.-à-d. qu'elle engendre la richesse des formes et des contenus de la production la plus diversifiée qui soit de tous côtés, lui soumettant tous les côtés de la nature50.

En somme, le temps de travail nécessaire économisé qui se trouve converti en surtravail dans les branches de production où s'opère une intensification de la production - soumission réelle - a pour contrepartie une conversion du temps de travail qui est libéré socialement dans cette branche de la production et qui permet,

50 Karl Marx, Manuscrits de 1857-1858 («Grundrisse »), t. 2, op. cit., p. 258. 
ce faisant, une extension du capital à de nouvelles sphères de la pratique sociale - soumission formelle -, et ainsi de suite. Voilà qui permet de rendre compte, d'un point de vue historique, de l'élargissement progressif du mode de production capitaliste à de nouvelles formes de pratiques sociales : pratiques agricoles, pratiques artisanales, professions libérales et secteur des services, activités scientifiques et technologiques, etc. Or, l'extension du capital à cette dernière forme d'activité et son développement intensif à travers la spécialisation du métier d'ingénieur entrouvrent toutefois un tout nouveau développement qui correspond cette fois à la soumission virtuelle de la pratique sociale au capital.

En effet, si la soumission formelle renvoie chez Marx à l'intégration de pratiques sociales préexistantes - passées - au mode de production capitaliste, et que la soumission réelle renvoie aux bouleversements - au présent - de leurs modalités organisationnelles et techniques, la soumission virtuelle renvoie quant à elle à la capacité à déterminer et contrôler les formes à venir - futures - des pratiques de production et de consommation à travers l'invention de nouveaux objets d'usage et la création de nouveaux besoins. Par conséquent, la soumission virtuelle touche aux dimensions fondamentales de la vie humaine que nous avons abordées précédemment, à savoir la réflexivité qui caractérise l'activité humaine et les formes contingentes sous lesquelles elle s'incarne historiquement. Or, cette capacité à déterminer de nouveaux objets d'usage et de nouveaux besoins n'est pas seulement mobilisée par les grandes corporations dans le cadre des stratégies de contrôle qu'elles mettent en œuvre. Elle se trouve de plus en plus monopolisée par elles, ce qui implique une «monopolisation de la capacité à monopoliser ${ }^{51} »$. En effet, la centralisation de puissance et de capitaux sans précédent qui caractérise la grande corporation a constitué la condition de possibilité de la mise en place de laboratoires de recherche privés consacrés à l'invention de nouveaux objets d'usage brevetés qui nécessitent de lourds investissements en équipements de pointe et en main-d'œuvre qualifiée, de même que pour la réalisation d'études de marketing et de campagnes publicitaires systématiques. Comme le remarque David Noble, les corporations acquirent alors une capacité à «déterminer le futur $5^{2}$ ». Autrement dit, elles disposent désormais de la capacité à canaliser les possibilités et le devenir des inventions et des besoins sociaux dans certaines directions au détriment des autres.

\footnotetext{
${ }^{51}$ Nous fusionnons ici une expression de David Noble, « monopoly of monopolies » (America by design, op. cit., p. 85 et 87), et une expression de Michel Freitag, « l'institutionnalisation de la capacité à institutionnaliser ", qui vise chez ce dernier à désigner le pouvoir moderne (Dialectique et société, vol. 3 : Culture, pouvoir, contrôle. Les modes de reproduction formels de la société, Montréal, Liber, 2013, p. 383 sq.).

$5^{2}$ David Noble, America by design, op. cit., p. 3.
} 


\section{Conclusion}

La subsomption virtuelle de la pratique sociale au capital se rapporte à l'avènement du capitalisme avancé et au rôle névralgique qu'y joue désormais la grande corporation en tant que sujet central de l'économie. Elle renvoie à l'intégration de la production scientifique et technologique au mode de production capitaliste qui s'est opérée au fil du développement de la grande corporation, de la mise en place de stratégies monopolistiques fondées sur la production et la commercialisation d'inventions brevetées et sur la montée parallèle du métier d'ingénieur. Elle résulte de la soumission formelle et réelle de la production scientifique et technologique réalisée à travers le développement de la profession d'ingénieur qui s'est différenciée de façon verticale et horizontale sous un ensemble de spécialisations. Cela dit, la spécificité de la soumission virtuelle renvoie au fait qu'elle se rapporte à la mobilisation et au développement systématique de la réflexivité caractéristique de l'activité humaine, soit la production de nouvelles idées, comprise comme développement de nouveaux objets d'usages et de nouveaux besoins auxquels correspondent toujours de nouvelles pratiques de production et de consommation. En ce sens, elle touche à des dimensions fondamentales de l'activité humaine telle qu'elle se trouve dépeinte par Marx, c'est-à-dire comme activité qui relève à la fois de la volonté et de la conscience. Autrement dit, ce qui est en jeu avec la soumission virtuelle porte sur les formes sociohistoriques de la pratique humaine et, plus fondamentalement encore, sur l'historicité même de cette pratique.

Pour conclure, deux enjeux majeurs méritent d'être soulignés en ce qui concerne l'intégration de la production scientifique et technologique dans le cadre de la dynamique propre au capitalisme. D'un côté, cette intégration a évidemment des implications fondamentales quant aux finalités de cette forme d'activité. La recherche scientifique et technologique, lorsqu'elle est réalisée dans le giron de la grande corporation, se trouve subordonnée à la finalité propre au mode de production capitaliste, à savoir la valorisation du capital. Les recherches sont donc orientées en fonction du développement d'inventions brevetables, commercialisables et rentables. À cet égard, il semble que la forme la plus problématique qu'emprunte la production scientifique et technologique dans ce contexte renvoie à l'obsolescence programmée, qu'il s'agisse tout simplement d'opérer des modifications de formes afin de rendre des objets d'usage périmés avec le développement de nouveaux modèles ou encore d'intégrer volontairement des failles dans les composantes des biens produits en vue d'en limiter la durée de vie utile. D'un autre côté, ce que s'arrogent les grandes corporations qui misent sur une stratégie d'affaires fondée sur la production et la commercialisation d'inventions, c'est une capacité à déterminer les objets d'usage futurs qui viendront prendre place au sein de notre monde et dont nous ferons un usage social. C'est donc le monde que nous habitons, ce monde qui est rempli d'objets d'usage développés et façonnés par la main humaine au fil d'une histoire plurimillénaire, ce monde qui est meublé de multiples objets d'usage que nous employons sans cesse dans la vie de tous les jours, c'est ce monde, donc, qui résulte désormais de plus en plus de l'emprise qu'exerce le 
capital à l'endroit du développement de la science et de la technologie. Une prolifération d'objets d'usage nouveaux qui viennent à chaque fois bouleverser notre manière de vivre en société.

Comme on le voit, l'avènement d'un capitalisme dit « cognitif» semble bien loin d'annoncer la crise ou le dépassement du capitalisme. Compte tenu de la capacité à déterminer les objets d'usage futurs qui viendront prendre place au sein de notre monde, il ouvre plutôt un champ virtuellement illimité à la reproduction élargie du capital. 